\title{
Raman spectroscopy with a 1064-nm wavelength laser as a potential molecular tool for prostate cancer diagnosis: a pilot study
}

Felipe L. Magalhães, Alexei M. C. Machado, Eduardo Paulino Jr., Sangram K. Sahoo, Ana M. de Paula, Aloísio M. Garcia, Ishan Barman, Jaqueline S. Soares, Marcelo Mamede, 


\title{
Raman spectroscopy with a 1064-nm wavelength laser as a potential molecular tool for prostate cancer diagnosis: a pilot study
}

\author{
Felipe L. Magalhães, ${ }^{a}$ Alexei M. C. Machado, ${ }^{a, b}$ Eduardo Paulino Jr., ${ }^{a}$ Sangram K. Sahoo, ${ }^{c}$ Ana M. de Paula, ${ }^{c}$ \\ Aloísio M. Garcia, ${ }^{c}$ Ishan Barman, ${ }^{\mathrm{d}, \mathrm{e}}$ Jaqueline S. Soares, ${ }^{\mathrm{f}}$ and Marcelo Mamede ${ }^{\mathrm{a}, *}$ \\ ${ }^{a} F$ ederal University of Minas Gerais, School of Medicine, Belo Horizonte, Brazil \\ bontifical Catholic University of Minas Gerais, Graduate Program on Electrical Engineering, Belo Horizonte, Brazil \\ 'Federal University of Minas Gerais, Department of Physics, Belo Horizonte, Brazil \\ dJohns Hopkins School of Medicine, Department of Oncology, Baltimore, Maryland, United States \\ e Johns Hopkins University, Department of Mechanical Engineering, Baltimore, Maryland, United States \\ ${ }^{\mathfrak{f}}$ Federal University of Ouro Preto, Department of Physics, Campus Universitário Morro do Cruzeiro, Ouro Preto, Brazil
}

\begin{abstract}
Raman spectroscopy is widely used to investigate the structure and property of the molecules from their vibrational transitions and may allow for the diagnosis of cancer in a fast, objective, and nondestructive manner. This experimental study aims to propose the use of the 1064-nm wavelength laser in a Raman spectroscopy and to evaluate its discrimination capability in prostate cancer diagnosis. Seventy-four spectra from patients who underwent radical prostatectomy were evaluated. The acquired signals were filtered, normalized, and corrected for possible oscillations in the laser intensity and fluorescence effects. Wilcoxon tests revealed significant differences between the benign and malign samples associated with the deformation vibration characteristic of nucleic acids, proteins, and lipids. A classifier based on support vector machines was able to predict the Gleason scores of the samples with 95\% of accuracy, opening a perspective for the use of the 1064-nm excitatory wavelength in prostatic cancer diagnosis. @ 2018 Society of Photo-Optical Instrumentation Engineers (SPIE) [DOI: 10.1117/1.JBO.23.12.121613]
\end{abstract}

Keywords: Raman spectroscopy; prostate cancer; fluorescence.

Paper 180421SSR received Jul. 21, 2018; accepted for publication Oct. 17, 2018; published online Nov. 3, 2018.

\section{Introduction}

Prostate cancer is one of the most common types of cancer that affects men. The National Cancer Institute estimates that $\sim 11.2 \%$ of men will be diagnosed with prostate cancer at some point during their lifetime and $4.8 \%$ of all cancer deaths in 2018 will be due to prostate cancer in the United States. ${ }^{1}$ Many men with prostate cancer may be asymptomatic and, without screening, would never know they have the disease. The main purpose of screening for prostate cancer is to identify high-risk localized prostate cancer that can be successfully treated, thereby preventing the morbidity and mortality associated with advanced or metastatic prostate cancer. Nevertheless, the harms of the screening test and subsequent harms from diagnosis and treatment must be weighed. The histopathological analysis has an important role in the management decisions. The current standard reference in prostate cancer diagnosis is the histopathological Gleason grading system, ${ }^{2}$ the accuracy of which depends on the experience and interpretation skill of the pathologist. The Gleason score (GS) system was reviewed in 2014 by the International Society of Urological Pathology (ISUP), aiming to improve the correlation between the histopathological grading system and the prognostic grade groups. ${ }^{3}$ Therefore, straightforward techniques are continually being developed for helping the assessment of the aggressiveness and the stage of the disease.

\footnotetext{
*Address all correspondence to: Marcelo Mamede, E-mail: mamede.mm@
} gmail.com
Raman spectroscopy is a technique capable of providing chemical information about a biologic tissue sample, and is gaining wide recognition as an objective method for the diagnosis of diseases in tissues. ${ }^{4-7}$ It has been successfully tested in a variety of diseases in organs, such as breast, ${ }^{8-10}$ brain, ${ }^{11,12}$ gastrointestinal, ${ }^{13-15}$ and skin cancers. ${ }^{16-18}$ Furthermore, Raman spectroscopy has also been used to study prostatic adenocarcinoma, correlating Raman spectrum and the GS system. ${ }^{19-22}$ These studies have been performed using near-infrared laser sources, such as 785 and $830 \mathrm{~nm}$, aiming to improve Raman scattering intensity. However, at these wavelengths, biological tissues and common glass sampling exhibit strong fluorescence.

In the context of examining prostate malignancies, Patel and Martin $^{23}$ used Raman spectroscopy to characterize the transitional, central, and peripheral zones of normal prostates, revealing larger concentrations of DNA and RNA in the peripheral zone, as well as differences in the relative concentration of lipids and proteins between the three zones. Crow et al. ${ }^{20}$ and Stone et al. ${ }^{19}$ carried out a series of studies on prostatic tissue using Raman spectroscopy. The first publication of normal prostate spectra dates from 2002. ${ }^{24}$ In 2003, researchers showed the ability to differentiate prostate cancer into three categories (Gleason $<7$, Gleason $=7$, and Gleason $>7$ ) and from benign prostatic hyperplasia with $89 \%$ accuracy from in vitro analysis of frozen biopsies. ${ }^{19,20}$

In 2005 , the possibility of using fiber optics to perform the examination of urogenital tissues in vitro was demonstrated, opening a perspective for applying the technique in vivo during

$1083-3668 / 2018 / \$ 25.00$ (C) 2018 SPIE 
endoscopic procedures and biopsies. ${ }^{21}$ Statistical regression methods were used to correlate the scattering spectra obtained from the histological samples with the combination of the spectra of several pure biochemical compounds. An increase in the relative concentration of DNA, choline, and cholesterol was shown to be associated with malignancy. ${ }^{19,25-27}$

While the molecular specificity of Raman spectroscopy and its ability to make in vivo measurements should significantly reduce the number of unnecessary endoscopic biopsies and prevent biopsy-related costs, it is still to be incorporated for such clinical prostate procedures. A limiting factor of prior research efforts has been the presence of significant background and the associated noise contributions. The use of 1064-nm dispersive Raman spectroscopy provides a viable alternative that reduces intrinsic fluorescence and minimizes tissue's photochemical changes. ${ }^{28,29}$ The signal-to-noise ratio is improved using 1064-nm wavelength instead of $785 \mathrm{~nm} .{ }^{30}$ Thermal or photochemical changes are also reduced when using photons with lower energy, which are not mutagenic like the photons in the ultraviolet range that induces the formation of thymine dimers in DNA. However, the spectroscopic measurement of prostate tissue chemistry using a 1064-nm laser for histopathology is largely unexplored.

This proof-of-concept study aims to propose and investigate the use of 1064-nm laser Raman spectroscopy in the characterization of prostate cancer. We hypothesize that a higher wavelength laser is able to provide significant different spectra between benign and malign sample while keeping the fluorescence effect low.

\section{Methods and Materials}

\subsection{Participants}

The biopsies of prostatic parenchyma used in this study were composed of 37 specimens, 14 benign and 23 malign, obtained from eight patients of the Hospital of the Federal University of Minas Gerais, Brazil. Patients with suspected neoplastic involvement of the prostate gland (after negative biopsy) or with histopathological diagnosis of prostatic cancer who were referred to the surgical treatment or with signs of biochemical recurrence of cancer (prostate specific antigen elevation) were evaluated clinically, laboratorially, and by imaging modalities. This study used only samples from the prostate glands of patients undergoing radical prostatectomy. After inclusion in the research protocol, patients with histopathological diagnosis of prostate cancer were referred to radiology and nuclear medicine to perform the initial staging with conventional imaging studies. The Brazilian Ethics Committee approved the study and signed informed consent was obtained from all patients.

Following the staging phase, the patients had their prostate glands surgically removed and analyzed according to the Hospital's standard procedure. Prostatic fragments were collected from the remaining prostate glands by a pathologist for the analysis using Raman spectroscopy. These fragments were stored in formaldehyde until the moment of the signal acquisition. The main surgical specimen was sent for routine anatomopathological examination.

\subsection{Sample Preparation}

Considering that the prostate removed from a patient presents multiple diagnoses, such as normal prostatic parenchyma, benign prostatic hyperplasia, intraepithelial neoplasia, and several gradations of malignancy depending on the analyzed region, about 30 fragments were collected from each of the eight remaining prostates, using a punch of $1 \mathrm{~mm}$ in diameter. Fragments were collected to the right and left of the apex, base, and middle third of the prostate. This large number of fragments per patient was necessary as Raman scattering measurements from fragments presenting subsequent divergent histopathological diagnosis were excluded. In addition, only Raman scattering measurements from benign parenchyma biopsies that presented larger amount of glands than of stroma were forwarded to data processing. The Raman spectra measured from each biopsy were performed without prior knowledge of histopathological diagnosis. The data collected were stored for further processing according to the exclusion criteria described above, which aimed to ensure that the measured spectrum corresponded to the diagnosis defined by the pathologists. Each fragment was independently preserved in eppendorf containing formaldehyde solution and immediately prior to measurements the formaldehyde solution was replaced with $0.9 \%$ physiological solution. After the measurements, the fragments were again stored in formaldehyde solution for subsequent histopathological processing. Due to the possible contamination of the sample spectra by paraffin residues, as demonstrated by Faolain et al., ${ }^{31}$ samples were tested after one, two, and three cycles of dewaxing.

\subsection{Signal Acquisition}

To perform the scattering measurements, the specimens were positioned in the central region of the equipment between two glass coverslips (Fig. 1). The use of the 1064-nm laser reduces the intensity of the Raman scattering so that a power of $300 \mathrm{~mW}$ was applied on the samples, a value 100 times higher than the power used with the 785-nm laser. Only two measurements were performed on each fragment as a larger number of measurements would increase the temperature and biochemically damage the sample. All scatter data collection was blind, without the knowledge of the diagnosis contained in each sample.

A total of 74 scans were obtained using a spectral resolution of $0.4 \mathrm{~cm}^{-1}$ and $\sim 6 \mu \mathrm{m}$ in diameter at the focus of the laser beam. These parameters were adjusted in agreement with related published works using the 1064-nm laser in biological samples

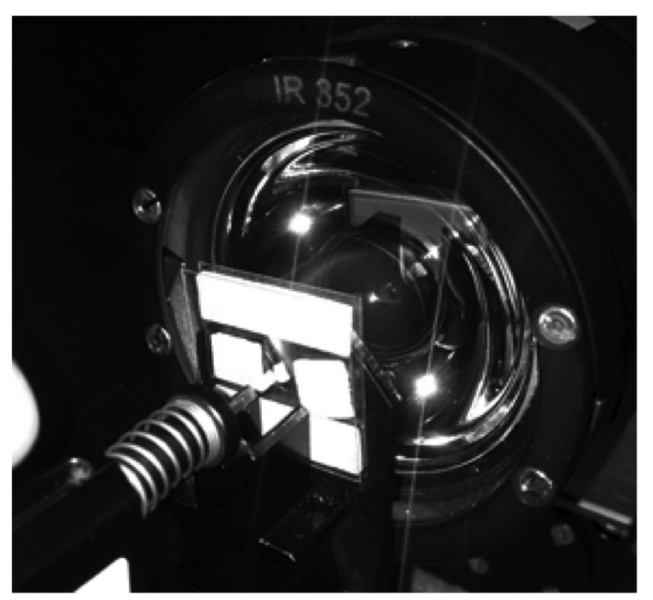

Fig. 1 Experimental setup with specimen positioning. 
as no published studies using this wavelength for the Raman spectroscopy of prostate samples could be found. A Bruker ${ }^{\circledR}$ VERTEX 70 FT-Raman spectrometer was used with the 1064-nm Nd:YAG laser as the excitation source. The calibration of the spectrometer followed three steps: the calibration with known lines of neon lamp, with silicon peak at $520 \mathrm{~cm}^{-1}$, and with known peaks of carbon nanotube and single-layer graphene. The Raman signal was collected by a germanium detector cooled by liquid nitrogen. The system uses a ROCKSOLID interferometer with the following acquisition parameters: spreading geometry of $180 \mathrm{deg}$, one point per the sample, and approximate sampling time of $60 \mathrm{~s}$.

\subsection{Sample Evaluation}

Following the measurements of the Raman scattering spectra, the fragments returned to the formaldehyde and underwent standard histopathological processing of the prostate gland. The collection of cuts was used to determine the exclusion criterion, i.e., the existence of more than one type of histopathological diagnosis per fragment. The slides were independently analyzed by two pathologists. A consensus was made in the case of disagreement regarding the diagnosis of the fragment. The overall concordance index among the pathologists was 0.51 (95\% of confidence interval, 0.37 to $0.65, p<0.001$ ), considered a moderate degree of concordance. For the diagnosis of normal glands, a Kappa of 0.83 (95\% of confidence interval, 1.00 to $0.51, p<0.001$ ) was considered excellent. The rate of sample utilization for data analysis was about $25 \%$ due to the exclusion criteria and losses during processing. From the set of 74 spectra, 28 were from benign samples, 12 had GS equal to 6, 28 had GS equal to 7, and 6 had GS equal to 8 .

\subsection{Data Preprocessing}

The set of 74 spectra of length $n=1851$ were initially filtered to remove high-frequency noise. The distribution of the noise was estimated by subtracting the average signal from the original ones. The probability distribution function of the differences was shown to be Gaussian based on hypothesis testing for skewness and kurtosis, ${ }^{32}$ with zero mean and standard deviation $s=1.19 \times 10^{-3}$. A wavelet-based filter implemented in the wavethresh $R$ package was used with cutoff level of 6 and the threshold set to $s \sqrt{2 \log n} .^{33}$ The cutoff level was experimentally determined so as to provide the largest number of significantly different variables as described in Sec. 3. All data preprocessing and statistical analysis were performed using the R software version 3.3.3.

The influence of the background composed of the coverslips and the hydration solution to which the fragments were submitted was additionally corrected, by subtracting their spectra from the sample. Two spectra of the glass coverslips, formaldehyde and physiological solution, were acquired, averaged, and filtered using the same parameters applied to the sample. The background spectrum was scaled so that its subtraction from the sample would not result in negative values.

Although the signals obtained with the 1064-nm laser present less fluorescence, an algorithm to remove baselines using fourth-degree polynomial regression provided by the baseline $\mathrm{R}$ package was applied. The resulting spectra were normalized based on the standard normal variate method $^{34}$ to eliminate influences from possible oscillations in the laser intensity.

\subsection{Data Analysis}

The nonparametric test of Wilcoxon was applied to the range of Raman shifts from 700 to $1800 \mathrm{~cm}^{-1}$ (571 measurements). This range (fingerprint) has shown to be of interest in the characterization of malignant tissues. ${ }^{22}$ The measurements were considered statistically different at the $p$-value level of 0.05 . The potential of the Raman spectroscopy using 1064-nm laser to discriminate between benign and malignant samples was additionally evaluated with the aid of support vector machine (SVM) classifiers. ${ }^{35}$ The svm procedure of the R package e1071 was used to implement a binary classifier to differentiate between malignant and benign, as well as a multiclass classifier to predict the GS of the specimens.

\section{Results}

The spectra of the saline solution, formaldehyde, and glass coverslips used in the preparation of the samples are shown in Fig. 2. The Raman spectroscopy of the coverslips produced a broad peak in the region of $1100 \mathrm{~cm}^{-1}$, which could potentially impair the identification of peaks generated by the fragments in this region. Therefore, the same coverslips were used in the experiments so that their influence would be minimized. The spectral analysis of the saline solution produced two broad scattering peaks in the region of interest, the first close to $800 \mathrm{~cm}^{-1}$ and the other at $1650 \mathrm{~cm}^{-1}$. The peak at $800 \mathrm{~cm}^{-1}$ is practically nonexistent in the scattering spectra of the fragments, whereas the $1650-\mathrm{cm}^{-1}$ peak coincides with a peak in the fragments' spectra. A peak at $1100 \mathrm{~cm}^{-1}$ was observed in the spectrum of the formaldehyde. As it was diluted in water and the peak at $800 \mathrm{~cm}^{-1}$ characteristic of the saline solution was not observed in the fragments, this suggests that the dilution process was sufficient to significantly reduce the presence of the formalin in the fragments.

Figure 3 shows the mean spectra of the benign and malignant groups after noise filtering, background removal, normalization, and baseline correction. As can be seen, the average spectrum of the malignant sample, shown in gray, presents higher values in almost all the fingerprint range. The local minimum at $800 \mathrm{~cm}^{-1}$ is a consequence of the subtraction of the saline solution

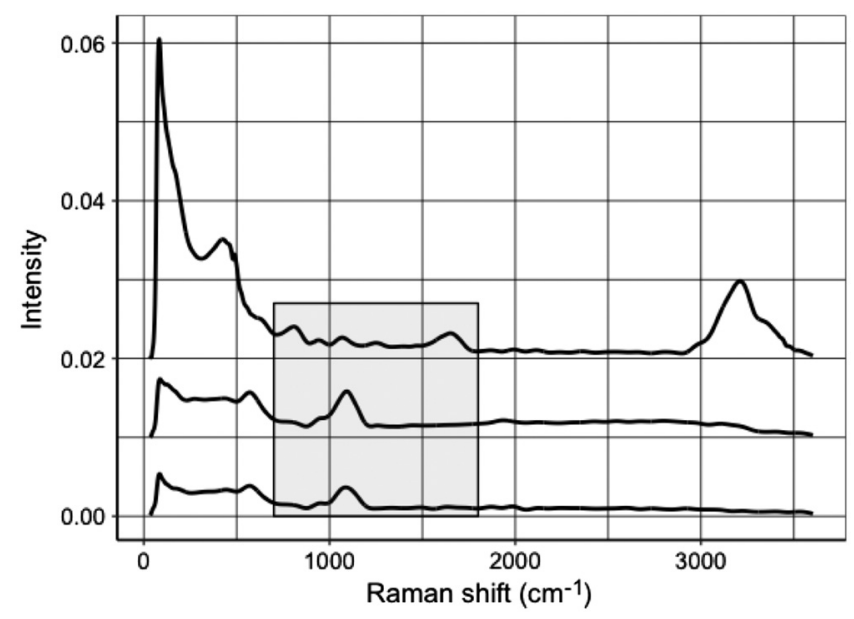

Fig. 2 Average filtered spectra of the physiological solution (top), formaldehyde (middle), and glass coverslips (bottom). The spectra are offset manually for visual clarity. The fingerprint region from 700 to $1800 \mathrm{~cm}^{-1}$ is shown in light gray. 


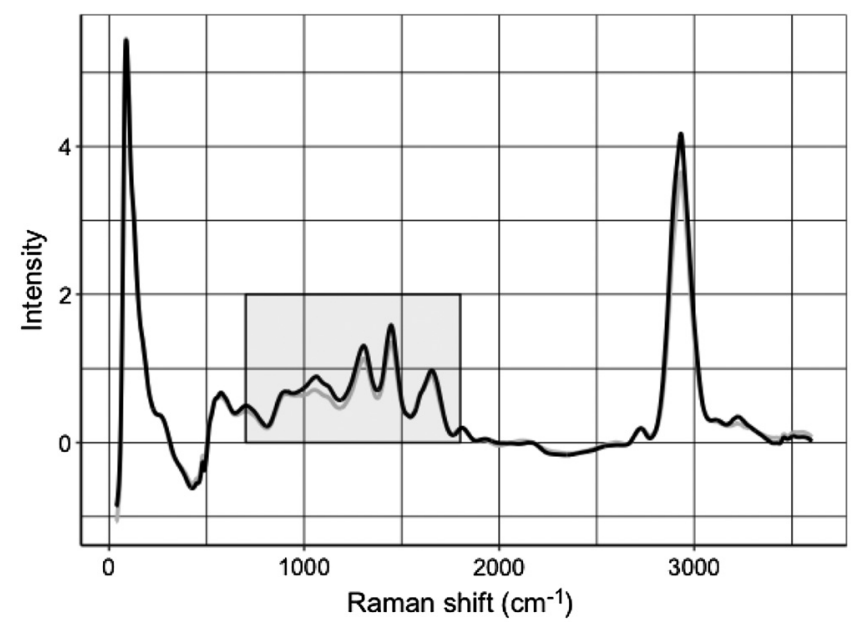

Fig. 3 Average spectra of the benign (gray) and malignant (black) samples. The fingerprint region from 700 to $1800 \mathrm{~cm}^{-1}$ is shown in light gray.

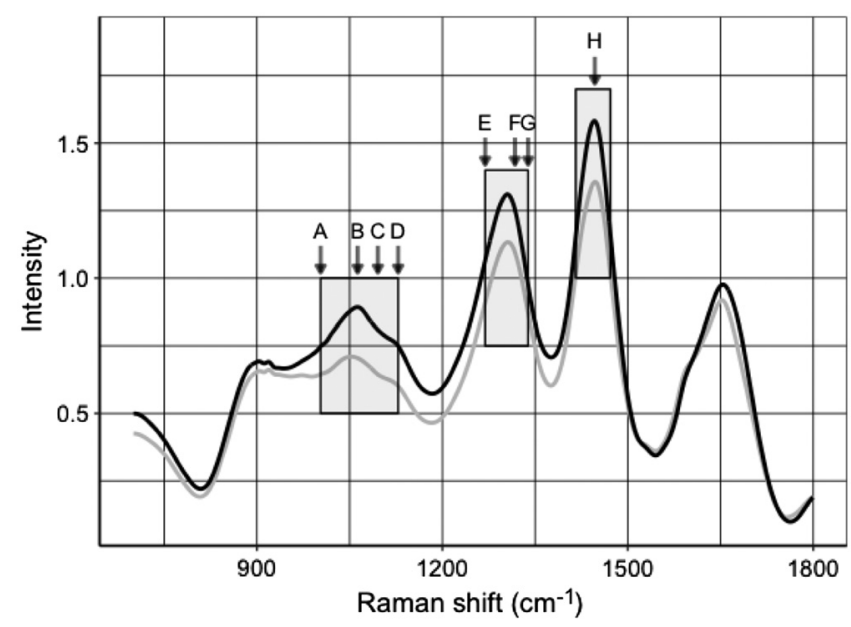

Fig. 4 Average spectra of the benign (gray) and malignant (black) samples in the fingerprint region. The most significant peaks are shown in light gray with key points labeled from $A$ to $H$ (see Table 1). spectrum, showing that the presence of preparation materials in the fragments was minimized.

Figure 4 shows the average spectra for both samples in the fingerprint region. The Wilcoxon test revealed significantly different signal magnitudes in the range from 980 to $1491 \mathrm{~cm}^{-1}$ (266 measurements), which encompasses eight shift points that have been identified in the literature as characteristic of prostate cancer (A to H). These points are shown in Table 1, with corresponding $p$-values and effect sizes.

Machine learning techniques were used to design a classifier able to discriminate between benign and malignant samples. A binary SVM was trained using a Gaussian kernel with gamma = 0.25 and cost $=1$. These values were determined with the aid of a grid searching process over the parameter space, performed by the tune.svm function of the e1071 package. The classifier used the eight measurements described in Table 1, taken at the Raman shifts of 1003, 1062, 1096, 1125, 1269, 1317, 1338, and $1447 \mathrm{~cm}^{-1}$. A leave-one-out cross-validation testing procedure resulted in $96 \%$ of accuracy. From the 74 samples, only three benign samples were misclassified as malignant.

A second classifier based on SVMs was designed to predict the GS of the samples. In this case, six binary SVMs using Gaussian kernel, gamma $=0.25$, and $\operatorname{cost}=1$ were trained to classify between each pair of classes taken from benign, $\mathrm{GS}=6, \mathrm{GS}=7$, and $\mathrm{GS}=8$. A voting strategy was used to determine the winner class, i.e., the sample was assigned to the class that was chosen by the largest number of SVMs. The classifier obtained an overall accuracy of 95\%. Table 2 shows the confusion matrix for the tests, using leave-one-out cross validation.

\section{Discussion}

The use of 1064-nm laser is still underexplored as an excitatory source in the analysis of prostatic tissue. In fact, studies based on Raman spectroscopy for the diagnosis of prostate cancer favor the use of lower wavelengths: $532,{ }^{39} 633,{ }^{37,38} 785,{ }^{21-23} 830,{ }^{19}$ and $832 \mathrm{~nm}^{20}$ The surface enhanced Raman spectroscopy (SERS) has also been applied to the analysis of blood serum to differentiate between prostate cancer and benign prostatic hyperplasia, using a 633-nm He-Ne laser. ${ }^{40,41}$ However, studies based on the coherent anti-Stokes Raman spectroscopy (CARS)

Table 1 Results of the Wilcoxon test showing the significantly different regions in the range of 700 to $1800 \mathrm{~cm}^{-1}$, labeled from A to $\mathrm{H}$ (see Fig. 4 ). References of concordant works are given in the first column. The vibration and molecular description is extracted from Kast et al. ${ }^{36}$

\begin{tabular}{lccccc} 
Label & Shift $\left(\mathrm{cm}^{-1}\right)$ & Vibration & Biomolecule & $P$-value & Effect size \\
\hline $\mathrm{A}^{21,32,37}$ & 1000 to 1003 & Symmetric ring breathing & Protein (phenylalanine) & 0.018 \\
$\mathrm{~B}^{32}$ & 1062 & $\mathrm{C}-\mathrm{C}$ stretch & Lipid & 0.53 \\
$\mathrm{C}^{21,37}$ & 1090 to 1100 & $\mathrm{O}-\mathrm{P}-\mathrm{O}$ & Lipid/phospholipid DNA backbone & 0.001 & 0.59 \\
$\mathrm{D}^{21,32,37,38}$ & 1125 to 1132 & $(\mathrm{C}-\mathrm{N})$ backbone $\mathrm{C}-\mathrm{C}$ stretch & Protein lipid/phospholipid & 0.002 \\
$\mathrm{E}^{32}$ & 1269 & $\mathrm{C}-\mathrm{N}$ stretch and $\mathrm{CH}_{2}$ wagging & Amide III & 0.46 \\
$\mathrm{~F}^{21,37}$ & 1317 to 1318 & & Lipid/phospholipid DNA/RNA & 0.014 \\
$\mathrm{G}^{37}$ & 1338 & & DNA/RNA & 0.71 \\
$\mathrm{H}^{21,37,38}$ & 1447 to 1450 & $\mathrm{CH}_{2}$ deformation & DNA/RNA protein lipid & 0.019 \\
\hline
\end{tabular}


Table 2 Confusion matrix and performance metrics achieved by the GS predictor.

\begin{tabular}{lcccc} 
& \multicolumn{4}{c}{ Diagnosis } \\
\cline { 2 - 5 } Predicted & Benign & GS $=6$ & GS $=7$ & GS $=8$ \\
\hline Benign & 27 & 0 & 0 & 0 \\
GS $=6$ & 0 & 11 & 0 & 0 \\
GS $=7$ & 1 & 1 & 28 & 2 \\
GS $=8$ & 0 & 0 & 0 & 4 \\
N. Spectra & 28 & 12 & 28 & 6 \\
Sensitivity & 0.96 & 0.92 & 1.00 & 0.67 \\
Specificity & 1.00 & 1.00 & 0.91 & 1.00 \\
Accuracy & & & 0.95 & \\
\hline
\end{tabular}

technique already adopt the 1064-nm wavelength as the Stokes beam with accurate results. ${ }^{42}$

The range from 700 to $1800 \mathrm{~cm}^{-1}$ is the region of the spectrum investigated by the majority of the published Raman spectroscopy applications on prostate cancer. The most evident peaks are found in $1000 \mathrm{~cm}^{-1}$ (phenylalanine ring breathing), 1200 to $1350 \mathrm{~cm}^{-1}$ (overlapping of the amide III, $-\mathrm{CH}_{2}-$ "wagging" and - $\mathrm{CN}-$ "stretch" peaks present mainly in lipids and nucleic acids), $1450 \mathrm{~cm}^{-1}\left(-\mathrm{CH}_{2}\right.$ deformation vibration present in nucleic acids, proteins, and lipids), and in the region of 1600 to $1700 \mathrm{~cm}^{-1}$ ( $\mathrm{C}=\mathrm{O}$ stretch, amide $\mathrm{I}$, present in nucleic acids and proteins). These peaks have been detected mostly by studies that used 633- and 785-nm dispersive systems in prostatic parenchyma, as reviewed by Kast et al. ${ }^{36}$

In the experiments performed with the 1064-nm laser, significant differences were found in the 1000 to $1450 \mathrm{~cm}^{-1}$ range, which are in accordance with previous reported results associated to lipids, nucleic acids, and proteins, among other biomolecules, as described in Table 1. Nevertheless, the experiments were not able to detect differences in the regions of 719 to $729 \mathrm{~cm}^{-1}$ and 781 to $786 \mathrm{~cm}^{-1}$, associated with DNA, RNA, and phospholipids, which were detected by other studies. ${ }^{21-23,37}$ In addition, we could not reproduce the findings of Crow et al., ${ }^{21}$ Taleb et al., ${ }^{37}$ and Patel and Martin, ${ }^{23}$ which reported differences in the range of 1657 to $1667 \mathrm{~cm}^{-1}$ associated with amide I.

The potential of the Raman spectroscopy to discriminate between benign and malignant samples as well as to predict the stage of the disease has been investigated since 2003 when Crow et al. ${ }^{20}$ applied linear discriminant analysis to classify a set of spectra from prostate biopsies. The study achieved $89 \%$ of accuracy for the Gleason prediction and $92 \%$ of accuracy for the classification into benign and malignant classes. Taleb et al. ${ }^{37}$ examined a set of 30 cells using partial leastsquares discriminant analysis and were able to correctly classify the sample into malignant and benign with $100 \%$ of accuracy. Discriminant function analysis associated with principal component analysis (PCA) was used by Devpura et al. ${ }^{22}$ to classify between cancerous and benign tissue samples with $91 \%$ of accuracy. PCA and SVM were used by Wang et al. ${ }^{38}$ to classify the spectra of 50 patients into two groups according to their
GS $(<=7$ and $>7)$, achieving $88 \%$ of sensitivity, specificity, and accuracy.

In the experiments using a 1064-nm excitatory source, we were able to classify the prostate samples into malignant and benign with $96 \%$ of accuracy, and to predict their GSs with $95 \%$ of accuracy. The choice for a machine-learning method such as the SVM incorporates the ability to discriminate nonlinearly separable classes that are not characteristic of other multivariate analysis such as the LDA. The specificity of the method was consistently high, with average of $98 \%$. The sensitivity varied from $67 \%$ to $100 \%$, with an average of $89 \%$. The lowest sensitivity of $67 \%$ was obtained for the GS $=8$ class, due to the fact that this was a reduced subset of six spectra. Nevertheless, the high accuracy of the results is encouraging and should motivate a deeper investigation on alternative wavelengths for the Raman spectroscopy of neoplastic tissues.

\section{Conclusion}

In this study, we have proposed and evaluated the application of the 1064-nm laser in Raman spectroscopy as an alternative to the 785-nm modality. In a set of experiments using benign and malignant fragments of the prostate parenchyma, the method was able to reveal differences in the spectra, opening a perspective for its use in cancer diagnosis. Raman spectroscopy using the 1064-nm excitatory laser allows for the evaluation of the scattering from different molecular compounds present in the prostatic tissue, in addition reducing the effect of fluorescence.

Subsequent experiments with a larger sample cohort will be pursued to confirm the method's ability to discriminate between groups and to provide the accurate GS for a given specimen. A larger sample set and repeated scans should increase the signalto-noise rate as well as the statistical power of the analysis. In addition to label-free Raman measurements, the use of SERS probes may permit precise visualization of tumor margins in vivo along with the characterization of microscopic tumor invasion. A more advanced perspective of application in prostatic tissue analysis would be the use of the CARS technique that requires a shorter signal integration time and could increase the sensitivity of the method. Ultimately, a 2-D mapping of histopathological sections should be output helping the pathologist to more accurately and objectively determine the prognostic groups of the patients from quantitative information of the biochemical composition of the prostatic tissue.

\section{Disclosures}

The authors declare having no financial interests in the manuscript and no other potential conflicts of interest to disclose.

\section{Acknowledgments}

We thank Marcos A. Pimenta who kindly provided the Raman Laboratory infrastructure at UFMG, where Raman spectroscopy measurements were performed. J.S.S. acknowledges financial support from CNPq, CAPES, and FAPEMIG.

\section{References}

1. Surveillance, Epidemiology, and End Results Program, National Cancer Institute, https://seer.cancer.gov/.

2. M. Goodman et al., "Frequency and determinants of disagreements and error in Gleason scores: a population-based study of prostate cancer," Prostate 72, 1389-1398 (2012).

3. S. Loeb, F. Montorsi, and J. Catto, "Future-proofing Gleason Grading: what to Call Gleason 6 Prostate Cancer?" Eur. Urol. 68, 1-2 (2015). 
4. Y. Ozaki, "Medical application of Raman spectroscopy," Appl. Spectrosc. Rev. 24, 259-312 (1988).

5. E. B. Hanlon et al., "Prospects for in vivo Raman spectroscopy," Phys. Med. Biol. 45, R1-R59 (2000).

6. A. Mahadevan-Jansen and R. Richards-Kortum, "Raman spectroscopy for the detection of cancers and precancers," J. Biomed. Opt. 1, 31-70 (1996).

7. C. J. Frank, R. L. McCreery, and D. C. Redd, "Raman spectroscopy of normal and diseased human breast tissues," Anal. Chem. 67, 777-783 (1995).

8. R. E. Kast et al., "Raman spectroscopy can differentiate malignant tumors from normal breast tissue and detect early neoplastic changes in a mouse model," Biopolymers 89, 235-241 (2008).

9. A. S. Haka et al., "In vivo margin assessment during partial mastectomy breast surgery using Raman spectroscopy," Cancer Res. 66, 3317-3322 (2006).

10. I. Barman et al., "Application of Raman spectroscopy to identify microcalcifications and underlying breast lesions at stereotactic core needle biopsy," Cancer Res. 73, 3206-3215 (2013).

11. A. Beljebbar et al., "Ex vivo and in vivo diagnosis of C6 glioblastoma development by Raman spectroscopy coupled to a microprobe," Anal. Bioanal. Chem. 398, 477-487 (2010).

12. M. Kirsch et al., "Raman spectroscopic imaging for in vivo detection of cerebral brain metastases," Anal. Bioanal. Chem. 398, 1707-1713 (2010).

13. M. S. Bergholt et al., "Characterizing variability in in vivo Raman spectra of different anatomical locations in the upper gastrointestinal tract toward cancer detection," J. Biomed. Opt. 16, 37003 (2011).

14. M. S. Bergholt et al., "Fiber-optic Raman spectroscopy probes gastric carcinogenesis in vivo at endoscopy," J. Biophotonics 6, 49-59 (2013).

15. A. Molckovksy et al., "Diagnostic potential of near-infrared Raman spectroscopy in the colon: differentiating adenomatous from hyperplastic polyps," Gastrointest. Endosc. 57, 396-402 (2003).

16. M. Gniadecka et al., "Melanoma diagnosis by Raman spectroscopy and neural networks: structure alterations in proteins and lipids in intact cancer tissue," J. Invest. Dermatol. 122, 443-449 (2004).

17. P. J. Caspers et al., "Combined in vivo confocal Raman spectroscopy and confocal microscopy of human skin," Biophys. J. 85, 572-580 (2003).

18. H. G. Edwards et al., "Potential applications of FT-Raman spectroscopy for dermatological diagnostics," J. Mol. Struct. 347, 379-387 (1995).

19. N. Stone et al., "The use of Raman spectroscopy to provide an estimation of the gross biochemistry associated with urological pathologies," Anal. Bioanal. Chem. 387, 1657-1668 (2007).

20. P. Crow et al., "The use of Raman spectroscopy to identify and grade prostatic adenocarcinoma in vitro," Br. J. Cancer 89, 106-108 (2003).

21. P. Crow et al., "Assessment of fiberoptic near-infrared Raman spectroscopy for diagnosis of bladder and prostate cancer," Urology $\mathbf{6 5}$ 1126-1130 (2005).

22. S. Devpura et al., "Detection of benign epithelia, prostatic intraepithelial neoplasia, and cancer regions in radical prostatectomy tissues using Raman spectroscopy," Vib. Spectrosc. 53, 227-232 (2010).

23. I. I. Patel and F. L. Martin, "Discrimination of zone-specific spectral signatures in normal human prostate using Raman spectroscopy," Analyst 135(12), 3060-3069 (2010).
24. N. Stome et al., "Near-infrared Raman spectroscopy for the classification of epithelial pre-cancers and cancers," J. Raman Spectrosc. 33(7), 564-573 (2002).

25. J. R. Krycer and A. J. Brown, "Cholesterol accumulation in prostate cancer: a classic observation from a modern perspective," Biochim. Biophys. Acta 1835, 219-229 (2013).

26. K. L. Zakian et al., "1H magnetic ressonance spectroscopy of prostate cancer: biomarkers of tumor characterization," Cancer Biomarkers 4, 263-276 (2008).

27. A. J. Breeuwsma et al., "In vivo uptake of [11C]choline does not correlate with cell proliferation in human prostate cancer," Eur. J. Nucl. Med. Mol. Imaging 32, 668-673 (2005).

28. A. L. Chad, W. Huawen, and W. Yang, "Tissue measurement using 1064 nm dispersive Raman spectroscopy," Proc. SPIE 8572, 857212 (2013).

29. A. Nijssen et al., "Discriminating basal cell carcinoma from perilesional skin using high wave-number Raman spectroscopy," J. Biomed. Opt. 12, 034004 (2007).

30. Y. K. Min et al., "1064 nm near-infrared multichannel Raman spectroscopy of fresh lung tissues," J. Raman Spectrosc. 36, 73-76 (2005).

31. O. Faolain et al., "A study examining the effects of tissue processing on human tissue sections using vibrational spectroscopy," Vib. Spectrosc. 38, 121-127 (2005).

32. A. Rencher, Methods of Multivariate Analysis, John Wiley \& Sons, New York (2002).

33. F. Ehrentreich and L. Summchen, "Spike removal and denoising of Raman spectra by wavelet transform methods," Anal. Chem. 73, 4364-4373 (2001)

34. K. H. Liland, A. Kohler, and N. K. Afseth, "Model-based pre-processing in Raman spectroscopy of biological samples," J. Raman Spectrosc. 47, 643-650 (2016).

35. C. Cortes and V. Vapnik, "Support-vector networks," Mach. Learn. 20(3), 273-297 (1995).

36. E. Kast et al., "Emerging technology: applications of Raman spectroscopy for prostate cancer," Cancer Metastasis Rev. 33, 673-693 (2014).

37. A. Taleb et al., "Raman microscopy for the chemometric analysis of tumor cells," J. Phys. Chem. B 110, 19625-19631 (2006).

38. L. Wang et al., "Raman spectroscopy, a potential tool in diagnosis and prognosis of castration-resistant prostate cancer," J. Biomed. Opt. 18(8), 087001 (2013).

39. M. Tollefson et al., "Raman spectral imaging of prostate cancer: can Raman molecular imaging be used to augment standard histopathology?" BJU Int. 106, 484-488 (2009).

40. M. Li et al., "Ultrahigh affinity Raman probe for targeted live cell imaging of prostate cancer," Chem. Sci. 7, 6779-6785 (2016).

41. N. Chen et al., "Surface-enhanced Raman spectroscopy of serum accurately detects prostate cancer in patients with prostate-specific antigen levels of 4-10 ng/mL," Int. J. Nanomed. 12, 5399-5407 (2017).

42. M. Potcoava et al., "Raman and coherent anti-Stokes Raman scattering microscopy studies of changes in lipid content and composition in hormone-treated breast and prostate cancer cells," J. Biomed. Opt. 19(11), 111605 (2014).

Biographies for the authors are not available. 